die von Ernest Cushing Richardson 1 . Heikels Edition hat nicht zu einer neuen englischen Übersetzung angeregt.

Das gilt auch für das Französische. Denn hier gibt es nur die alte Übersetzung von Louis Cousin ${ }^{2}$.

\title{
Die Kapiteleinteilung
}

Die Kapiteleinteilung in den Handschriften weicht oft von der der Editionen ab. Um nicht Verwirrung zu stiften, wurde auch in der vorliegenden Ausgabe die herkömmliche Einteilung beibehalten. Die Abweichungen der Handschriften werden hier zusammengestellt.

S. 15, 14 Kap. 2 V; 15, 20 Kap. 3 V; 16, 5 Kap. J; 16, 8 kein

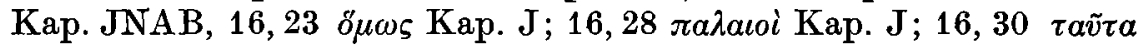
Kap. 4 V; 17, 3 kein Kap. Mss; 17, 10 kein Kap. JNAB; 17, 29 kein

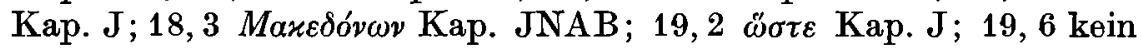

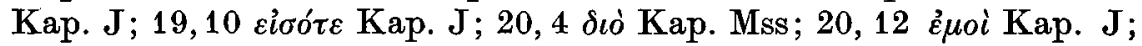

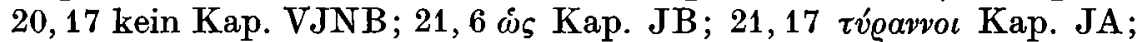

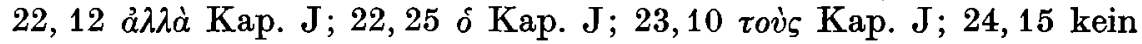

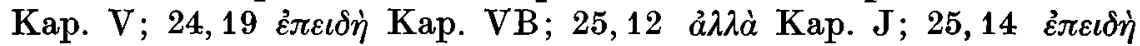

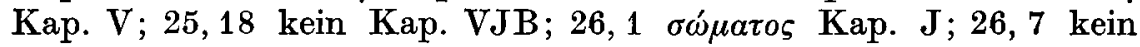

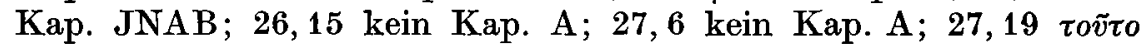

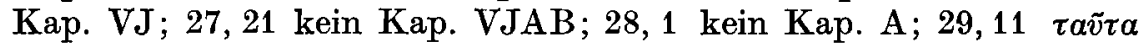

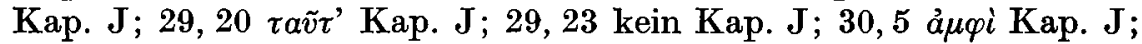

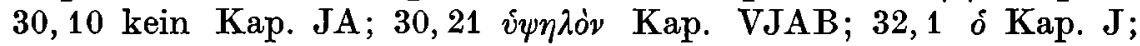

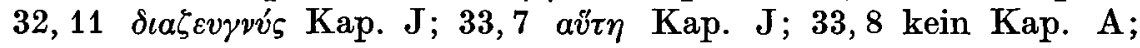

1 S. Bagster, The Greek Ecclesiastical Historians of the first six centuries, vol. 1: The Life of the Blessed Emperor Constantine, London 1845. - Eine Ưberarbeitung dieser Übersetzung von E. C. Richardson erschien Oxford und New York 1890 in: A select Library of the Nicene and Post-Nicene Fathers of the Christian Church NS 1, 481-559. - Eine englische Auswahlübersetzung: Palestine Pilgrims' Text Society vol. 15: Eusebius, Pamphili, Bishop of Caesarea. The Churches of Constantine at Jerusalem. Being translated from Eusebius ... London $18918^{\circ}$.

2 1627-1707. Vgl. Roman d'Amat, Dict. de Biogr. franç. 9, 1962, 1067 f. -

Histoire de l'Eglise écrite par Eusèbe Evêque de Cesarée. Traduite par Monsieur Cousin, Président en la Cour des Monnoyes. Dediée au Roi, à Paris, en la Boutique de Pierre Rocolet MDCLXXV, avec privilege du Roi. La vie de l'empereur Constantin, écrite par Eusèbe Evêque de Cesarée pp. 497-703. Es folgen Discours de l'empereur ... . und Harangue à la louange de l'empereur. - Die Kephalaia stehen nur vor den Kapiteln. Dem Band sind vorangestellt eine Epitre au Roy und ein ausgedehnter Avertissement. Es folgt ein Extrait du Privilege du Roi, unterschrieben: Registré sur le Livre de la Communauté le 1. Juin 1674. Signé D. Thierry, Syndic. Achevé d'imprimer pour la premiere fois le 2. Janvier 1675. Am Ende der Avertissement heißt es: „Je me suis servi du texte Grec que Monsieur de Valois l'un des plus savans hommes de nôtre siècle a corrigé ...." 
33,11 в̇лi Kap. B; 33,18 kein Kap. A; 35, 2 блтvixa Kap. J; 36,1 kein Kap. A; 36, 11 kein Kap. A; 37, 3 kein Kap. A; 37, 18 kein Kap. A; 38, 5 kein Kap. A; 38, 25 kein Kap. A; 39, 5 عiף' Kap. J;

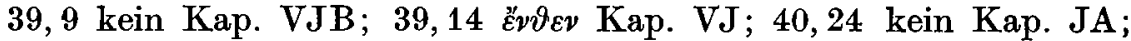



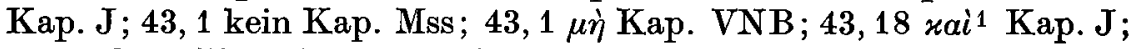
43, 23 kein Kap. J; 43, 30 ov Kap. J; 44, 20 kein Kap. J; 44, 23 ả @ó́a

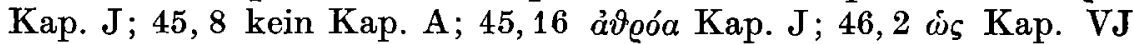
(V: Kap. 60); 46, 6 kein Kap. Mss; 47, 15 kein Kap. VNAB Hist Gel;

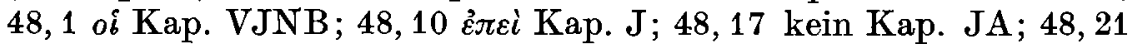

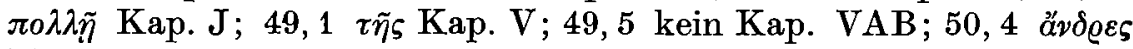

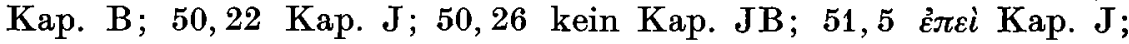
51, 11 kein Kap. A; 52, 4 a $\lambda \lambda$ ' Kap. VB; 52, 15 Kap. 11 V; 52, 23 kein

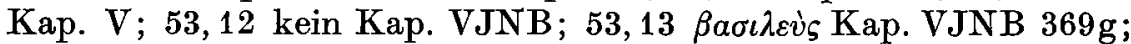
54, 1 kein Kap. JA; 55, 1 kein Kap. B; 55, 16 kein Kap. JNB; 56, 8 rovaexías Kap. J; 57, 1 oi Kap. J; 57, 10 kein Kap. A; 57, 19 kein



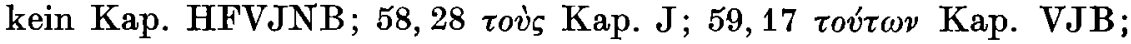
59, 19 kein Kap. VJB; 62,22 kein Kap. J; 63, 9 kein Kap. JNAB;

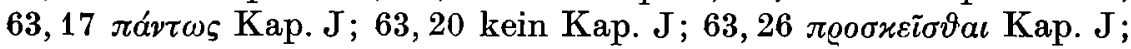
64, 3 kein Kap. J; 64, 9 ö $\mu \omega \varsigma$ Kap. 38 ; ; 64, 14 kein Kap. $b$; 64, 22 $\delta \sigma \alpha$ Kap. J; 65, 1 kein Kap. JA; 65, 24 kein Kap. J; 6617 kein Kap.

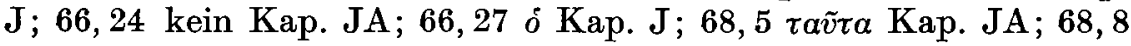

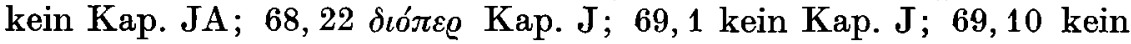
Kap. A; 69, 26 kein Kap. A; 70, 4 kein Kap. J; 70,11 kein Kap. A;

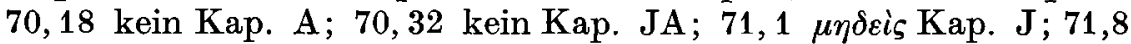
kein Kap. VJNA; 71, 12 à $\lambda \lambda \dot{\alpha}$ Kap. 58 V; 71,15 kein Kap. A; 72, 7


Kap. A; 74, 5 kein Kap. J; 74, 13 kein Kap. A; 75, 2 kein Kap. A;

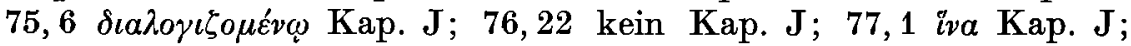
77, 24 rai Kap. J; 78,6 тò Kap. J; 78, 13 kein Kap. JA; 79, 3 kein Kap. A; 80, 22 oi Kap. J; 81, 8 oi Kap. JNB; 81, 18 oi Kap. J; 82,1

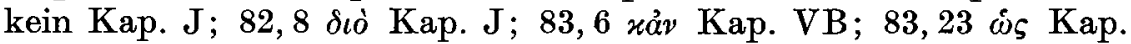
JNA; 84, 8 kein Kap. JB; 84, 13 光 $\eta$ Kap. J; 85, 6 kein Kap. NB; 85, 16 kein Kap. A; 85, 22 kein Kap. J; 86, 4 a $\lambda \lambda$ ' Kap. J; 86, 18

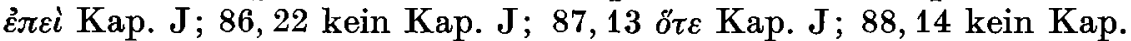

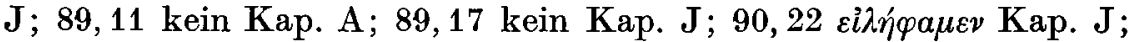

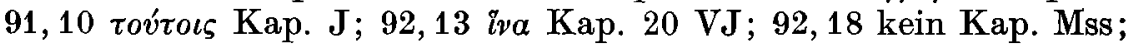

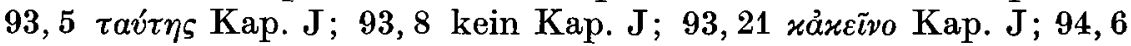
kein Kap. A; 94, 10 kein Kap. JA; 94, 18 kein Kap. A; 94, 24 kein

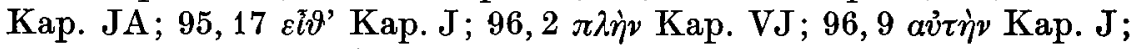
96,13 kein Kap. VJNBmg; 96, 16 ả $\lambda \lambda^{\prime}$ Kap. VJNB; 96, 19 kein Kap. Mss; 97, 1 kein Kap. J; 99, 3 kein Kap. B; 99, 25 kein Kap. A; 100, 2 kein Kap. J; 100, 5 kein Kap. J; 100, 7 or̃ Kap. J; 100, 27 kein Kap. 
JA; 101, 7 kein Kap. J; 102, 5 jádı Kap. J; 102, 30 kein Kap. J; 103, 11 rai Kap. VJNB; 103, 18 kein Kap. VJNB; 103, $21 \tilde{\omega} \delta \varepsilon$ Kap.

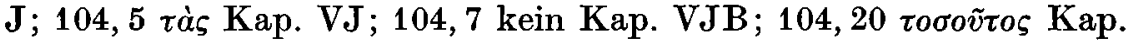

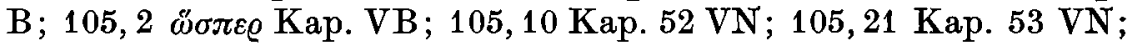

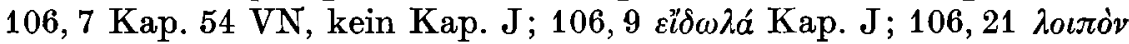



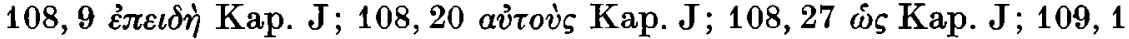

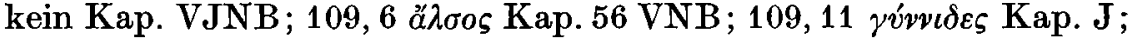
110, 1 Kap. 57 VN; 110, 12 a $\lambda \lambda$ ' Kap. J; 110, 16 Kap. 58 VN, kein

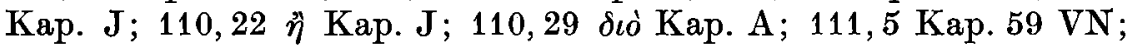
111, 9 xai Kap. J; 111, 24 Kap. 60 VN; 112, 9 Kap. V (61) JNB;


Kap. J; 114, 24 ह̇лi Kap. J; 115, 8 Kap. 63 VN; 116, 3 Kap. 64 VN;

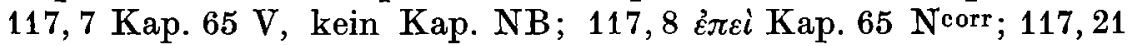
Kap. 66 VN; 118, 5 av่̇ทे Kap. J; 118, 17 kein Kap. V; 118, $23 \pi \lambda \dot{\nu}$

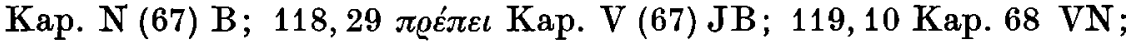

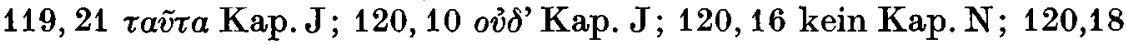

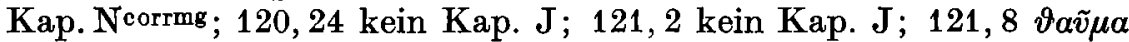
Kap. VNB 369g; 122, 1 oข̃ $\omega$ Kap. 7 VJNB; 122, 4 kein Kap. VJNB; 122, 14 oi Kap. J; 123, 22 kein Kap. J; 123, 26 d Kap. J; 125, 12

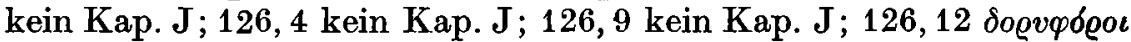

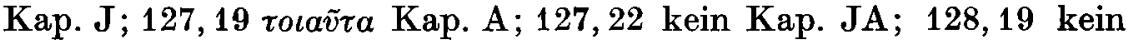

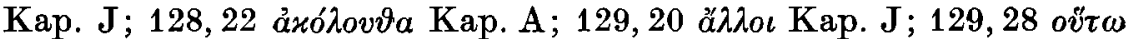

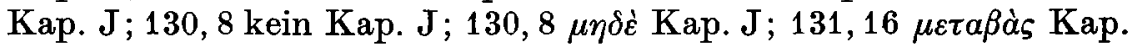
VJNB; 131, 27 ả $\lambda \lambda$ ' Kap. J; 131, 30 kein Kap. VJNB; 132, 2 ả $\lambda \lambda$ '

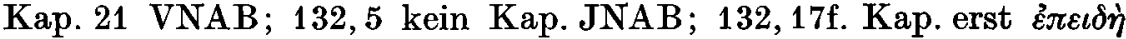

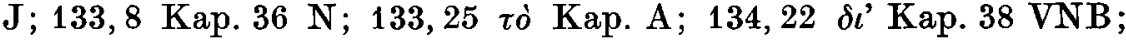

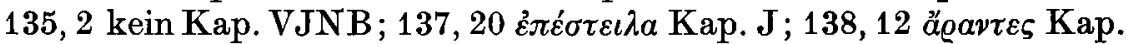
J; 139, 9 a $\lambda \lambda$ ' Kap. 45 VJNB; 140, 12 kein Kap. J; 140, 20 kein Kap.

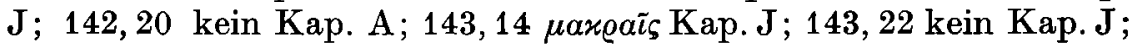
144, 2 Kap. 58 VJNcorr B; 144, 15 kein Kap. J; 145, 4 a $\lambda \lambda$ ' Kap. 61

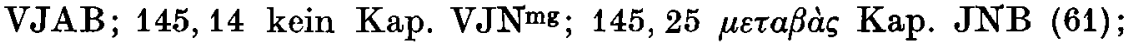
145, 26 kein Kap. VJNB; 146, 1 غ̇лi Kap. 62 NB; 146, $9 \delta$ Kap. 63

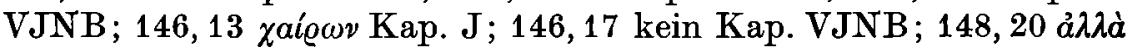
Kap. 68 VJNB; 149, 13 kein Kap. J; 149, 13 o Kap. J; 149, 21 kein Kap. VJN1B; 150, $1 \dot{\omega}$ Kap. 71 VJ ; 150, 1 tò Kap. B; 150, 7 kein Kap.

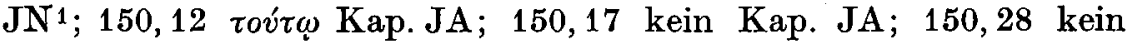
Kap. JA.

\section{Die Kephalaia}

Den einzelnen Büchern sind in den Handschriften als geschlossener Block Kapitelüberschriften vorangestellt (in A nur dem ersten Buch). Zusätzlich sind diese Kephalaia in $\mathrm{V}$ in teilweise variierender Form 